\title{
Recent developments and future of the GM/CA@APS X-ray crystallography user facility at the Advanced Photon Source
}

Michael Becker $^{1}$, Stephen Corcoran ${ }^{1}$, Dale Ferguson ${ }^{1}$, Mark Hilgart ${ }^{1}$, David J. Kissick ${ }^{1}$, Oleg Makarov $^{1}$, Craig M. Ogata ${ }^{1}$, Ruslan Sanishvili1, Sergey Stepanov ${ }^{1}$, Nagarajan Venugopalan ${ }^{1}$, Qingping $\mathrm{Xu}^{1}$, Shenglan $\mathrm{Xu}^{1}$, Robert F. Fischetti ${ }^{1}$, Janet L. Smith ${ }^{2}$

${ }^{1}$ GM/CA@APS, Argonne National Laboratory, Argonne, IL, ${ }^{2}$ Life Sciences Institute, University of Michigan, Ann Arbor, Ml

The General Medical Sciences and Cancer Institutes' structural biology facility at the Advanced Photon Source (GM/CA @ APS) operates a national user facility for crystallographic structure determination of biological macromolecules by X-ray diffraction, including two undulator beamlines - beamline 23IDB, and beamline 23IDD - with an emphasis on advancing challenging, high-impact projects. This poster gives an overview of recent developments, as well as long-term aims relevant to the planned APS upgrade. Updates at beamline 23IDB include (a) a Dectris Eiger detector was recently incorporated, and (b) the development of the second-order nonlinear optical imaging of chiral crystals (SONICC) system progresses with improved optics. Updates at beamline 23IDD include (a) a new high-capacity sample automounter has tripled the puck capacity to 18 , which is particularly helpful for remote operations, and (b) plans for compound reflective lens $(C R L)$ optics for X-ray focusing down to $\sim 1 \mu \mathrm{m}$ at beamline 23IDD are in the works, which will dovetail nicely with the planned upgrade of the APS ring. New software enhancements will also be discussed. Finally, proof-of-concept experiments in X-ray FEL-like, serial crystallography experiments have been performed for fixed and injector-based sample delivery systems, as well as for in situ crystallization screening experiments.

Acknowledgment: GM/CA@APS has been funded in whole or in part with Federal funds from the National Cancer Institute (ACB-12002) and the National Institute of General Medical Sciences (AGM-12006). This research used resources of the Advanced Photon Source, a U.S. Department of Energy (DOE) Office of Science User Facility operated for the DOE Office of Science by Argonne National Laboratory under Contract No. DE-AC02-06CH11357. The Eiger 16M detector was funded by an $\mathrm{NIH}-$ Office of Research Infrastructure Programs, High-End Instrumentation Grant (1S10OD012289-01A1). 